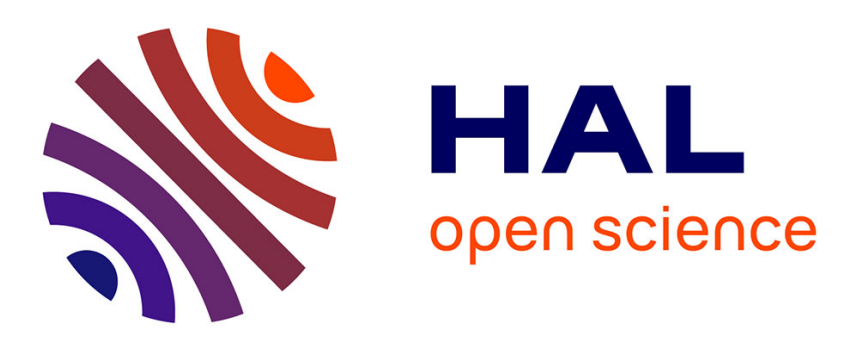

\title{
ÉTUDE DU COMPORTEMENT DES COMPOSITES À FIBRES DE VERRE/ÉPOXY EN CISAILLEMENT À GRANDE VITESSE DE DÉFORMATION
}

\author{
C. Chiem, Z. Liu, J. Ramousse
}

\section{- To cite this version:}

C. Chiem, Z. Liu, J. Ramousse. ÉTUDE DU COMPORTEMENT DES COMPOSITES À FIBRES DE VERRE/ÉPOXY EN CISAILLEMENT À GRANDE VITESSE DE DÉFORMATION. Journal de Physique Colloques, 1985, 46 (C5), pp.C5-535-C5-542. 10.1051/jphyscol:1985569 . jpa-00224802

HAL Id: jpa-00224802 https://hal.science/jpa-00224802

Submitted on 1 Jan 1985

HAL is a multi-disciplinary open access archive for the deposit and dissemination of scientific research documents, whether they are published or not. The documents may come from teaching and research institutions in France or abroad, or from public or private research centers.
L'archive ouverte pluridisciplinaire HAL, est destinée au dépôt et à la diffusion de documents scientifiques de niveau recherche, publiés ou non, émanant des établissements d'enseignement et de recherche français ou étrangers, des laboratoires publics ou privés. 
JOURNAL DE PHYSIQUE

Colloque C5, supplément au n8, Tome 46, ao0t 1985

page C5-535

ÉTUDE DU COMPORTEMENT DES COMPOSITES À FIBRES DE VERRE/ÉPOXY EN CISAILLEMENT A GRANDE VITESSE DE DÉFORMATION

\author{
C.Y'. Chiem, Z.G. Liu et J.P. Ramousse \\ Ecole Nationale Supérieure de Mécanique, Laboratoire des Sciences des \\ Matériaux de za Mécanique, Groupe DYNAMAST, 44000 Nantes, France
}

\begin{abstract}
Résumé - Cette étude du comportement au choc des composites à résine époxy utilise le principe des barres de Kolsky en torsion modifiées pour le cisaillement. Une description succincte de la méthode est faite. Elle est suivie de résultats expérimentaux avec une discussion sur les problèmes rencontrés au plan expérimental. Il semble que la vitesse de déformation critique autour de $2000 \mathrm{~s}^{-1}$ pose le problème le plus important au niveau du comportement aux grandes vitesses de défonmation des composites U.D. à matrice de résine. Des problèmes de délaminage généralisé, de cisaillement interlaminaire et d'élévation de température en cours de déformation à grande vitesse se sont posés.
\end{abstract}

Abstract - The principle of torsional Kolsky bars modified for simple shear tests are applied for this study on epoxy-resin and glass-fiber composites. A short description of the method is done. It is followed by discussions on problems encountered in the experimental results. It seems that a critical strain-rate around $2000 \mathrm{~s}^{-1}$ enhances an important point on the high-strainrate behaviour of U.D. composites with resin matrix ; Problems concerning generalized delamination, interlaminar shear and termperature increment during high strain-rate deformation are mentioned.

\title{
I - INTRODUCTION
}

Le comportement dynamique des matériaux composites soumis aux grandes vitesses de deformation reste un problème ouvert de grandes complexités théorique et expérimentale. F. Devries et F. Lene /1/ ont donné une analyse théorique par une schématisation du phénomène de pénétration en milieu composite. Au point de vue expérimental, C.y. Chiem et al./2/ ont classé dans une monographie des résultats assez complets du sujet. Dans les essais utilisant la technique des barres de Hopkinson, trois types d'essais sont intéressants à citer : compression, traction, cisaillement.

On ne propose, ici, que les essais par barres de Kolsky (ou de Hopkinson) en torsion. Cet article présente successivement la méthodologie, l'interprétation des premiers résultats et 1'aspect de I'endonmagement des matériaux composites suivants : composite I : tissu de verre équilibré / résine 470-36 ; composite II : tissu de verre équilibré / résine XD 8084-05.

\section{II - METHODOLOGIE DES BARRES DE KOLSKY FN TORSION}

2.1. Principe des barres de Kolsky en torsion

Le dispositif de Kolsky en torsion est décrit à plusieurs reprises nar de nombreux auteurs tels que Duffy et al. /3/, Chiem et al. /4,5,6/. Ainsi, nous ne faisons qu'un descriptif rapide de ce dispositif.

Un dispositif de Kolsky en torsion est généralement constitué d'une barre d'entrée, d'une barre de sortie et d'un système moteur qui sert à appliquer une prétorsion entre I'extrémité libre de la barre d'entrée et des mâchoires. Lors de l'ouverture des mâchoires obtenue par la rupture d'une vis de serrage, le couple enmagasiné se propage dans la barre d'entrée, se réfléchit partiellement et se transmet à travers 
l'échantillon dans la barre de sortie. Ia figure 1 présente ce dispositif d'essais.

Si v est la vitesse tangentielle sur la barre d'entrée, on a :

$$
\mathrm{v}=\mathrm{r} \cdot \mathrm{\omega}=2 \cdot \frac{\mathrm{T} / 2}{\mathrm{~J} \rho \mathrm{C}_{\mathrm{T}}} \cdot \mathrm{r}
$$

où $\mathrm{T}_{\mathrm{O}}$ est le couple emmagasiné, $\mathrm{J}$, le moment polaire de la section de la barre, $\rho$, la masse volumique du matériau de la barre et $C_{\text {Tr }}$ la vitesse de l'onde de torsion dans les barres. $\omega$ est la vitesse angulaire de fa barre. Compte tenu que $\left(\mathrm{T}_{\mathrm{O}}\right)_{\max }=\frac{\tau_{\mathrm{y}} \cdot \mathrm{J}}{\mathrm{r}}$ où $\tau_{\mathrm{y}}$ est la limite élastique du matériau de la barre,
on a :

$$
v_{\max }=\frac{\tau}{\rho C_{T}}
$$

Si I'on suppose que le rayon maximum de I'échantillon est égal à celui de la barre, la vitesse de déformation maximale est alors :

$$
\dot{\bar{\gamma}}_{\max }=\frac{\tau_{\mathrm{y}}}{\rho C_{\mathrm{T}} \ell_{\mathrm{L}}}
$$

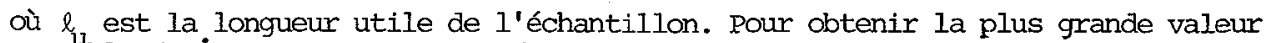
possible de $\dot{\gamma}_{\text {max }}$ il faut que $\tau / \rho C_{T}$ soit maximum pour les barres et que $\operatorname{lu}_{\mathrm{v}}$ soit minimum pour líéchantillon. C'est ainsi que nous avons choisi l'alliage d'aluminium 79 comme matériau des barres $\left(G=26800 \mathrm{MPa}, \rho=2700 \mathrm{~kg} / \mathrm{m}^{3}, C_{\mathrm{T}}=\checkmark \frac{\mathrm{G}}{\rho}=3100 \mathrm{~m} / \mathrm{s}\right)$.
Le rayon des barres est de $15 \mathrm{rm}$.

En ce qui concerne le traitement des signaux, on peut utiliser les signaux de l'onde réfléchie, et de 1'onde transmise; le premier type de signal donne la vitesse de déformation et la déformation, le deuxième donne la contrainte de cisaillement au niveau de l'échantillon. Ce procédé décrit en termes de couples d'entrée $T_{i}$ et de sortie $T_{t}$ (voir figure 2) se traduit par les formules suivantes :

- Ia contrainte de cisaillement au niveau de l'échantillon parallélépipédique est (cf. fig. 3) :

$$
\tau=\frac{T_{t}}{2 r_{m} h_{1} h_{2}}
$$

où $r_{m}$ est le rayon moyen de l'échantillon.

- La vitesse de déformation est calculée par la relation :

$$
\dot{\gamma}=\frac{2\left(\mathrm{~T}_{i}-\mathrm{T}_{t}\right)}{\mathrm{J} \rho \mathrm{C}_{\mathrm{T}}} \cdot \frac{\mathrm{r}_{\mathrm{m}}}{\mathrm{h}_{3}}
$$

Notons que $T_{i}-T_{t}=T_{r}\left(T_{r}=\right.$ couple de l'onde réfléchie)

- La déformation est dono obtenue par intégration de $\dot{\gamma}$ en fonction du temps.

\subsection{Définition de l'échantillon dans le premier lot d'essais}

Les composites qu'on nous a fournis sont des plaques (renfort en poids $70 \%$, tissu à 8 couches croisées à $90^{\circ}$ ). La figure 3 et le tableau 1 donnent la géométrie des échantillons. Les échantillons entaillés permettent d'avoir un endonmagement dans la zone de cisaillement. Le problème majeur dans les essais en cisaillement est la transmission du couple à l'échantillon. Des essais de fixation par collage ont montré qu'il y avait rupture au niveau de la couche externe du composite constituée de résine. Aussi, avons nous adopté le système d'encastrement (cf. fig. 4) renforcé par un collage avec une colle de haute performance (Araldite AW 106 et durcisseur HV $953 \mathrm{U})$.

\subsection{Intérêt de la méthodologie}

Lors de la propagation d'une onde de torsion, le matériau peut être considéré conme 
ébranlé progressivement lors du passage de l'onde. Ceci a un intérêt évident dans l'étude d'un matériau laminé constitué de tranches successives de fibres de verre et de résine.

Les vitesses de déformation obtenues varient de 100 à $5000 \mathrm{~s}^{-1}$, et les temps de montée de 1 'onde de 20 à $30 \mu \mathrm{s}$, selon la nature du matériau testé et le couple de prétorsion imposé.

Cette méthodologie permet d'effectuer des essais sur des matéxiaux anisotropes tels que monocristaux et composites à fibres. Dans ce cas, la géométrie des échantillons est parallélépipéđique. Ia direction de cisaillement étant une direction privilégiée du matériau.

\subsection{Interprétation des résultats}

Les essais de cisaillement dynamique permettent donc d'obtenir la vitesse de déformation et la contrainte dans l'échantillon en fonction du temps. Par intégration, on peut obtenir alors la déformation, d'où la courbe contrainte-déformation. Les premiers résultats ont été obtenus sur le composite à tissu de verre équ」libré à résine 470-36. La figure 5 montre les courbes "contrainte-déformation" obtenues sur trois types d'échantillon (fig. 3). On voit que la contrainte maximale ( $\tau_{\max }$ ) est fortement sensible à la vitesse de déformation, sauf pour les courbes suivaxhtes : $n^{\circ} 5$ (type I), $n^{\circ} 5,7$ (type II) et $n^{\circ} 5,7$ (type III) qui seront expliquées par 1'allure de 1 'endommagement dans le paragraphe III. La figure 6 présente $\tau_{\text {max }}$ en fonction de $\dot{\gamma}$. On constate une certaine linéarité pour les trois types d'échantixllons. Il semble que l'entaille n'influence pas fortement le comportement mécanique des échantillons. pour cette raison, nous poursuivrons les essais sur d'autres composites avec la forme d'échantillons du type III.

\section{III - EXPLICATION DU COMPORTEMENT ET ANALLYSE DE L'ENDOMMAGEMENT}

Les essais ont été effectués sur les composites I et II. Ia géométrie des échantillons est du type III.

\subsection{Résultats expérimentaux}

La figure 7 montre les courbes contrainte-déformation en dynamique et en quasi-statique. Chaque courbe est la moyenne de trois essais (soit 6 échantillons). Les contraintes maximum pour les composites testées ont presque les mêmes valeurs. La déformation de cisaillement pour le composite II est plus importante que celle du composite I. Ceci reste à expliquer par l'étude du comportement dynamique de ces deux résines pures. Il est à noter que les contraintes dans les essais quasi-statiques ne sont pas les contraintes maximum dans le composite à cause du collage insuffisant entre l'échantilion et les brides. On voit aussi une même allure d'augmentation de la contrainte maximum en fonction de la vitesse de déformation pour les deux composites, sauf dans les courbes $n^{\circ} 3$. Il convient d'analyser $1^{\prime}$ 'endommagement des échantillons pour expliquer ce phénomène.

\subsection{Analyse de l'endommagement}

Nous proposons dans le tableau 2 des schémas représentatifs de 1'endormagement correspondant aux courbes de la figure 7 . Nos essais n'ont jamais mené à la rupture dans la direction du cisaillement, mais à un endommagement avec fissuration de plus en plus important avec l'augmentation de $\dot{\gamma}$ et $\gamma$. Quand la vitesse de déformation dépasse une valeur critique $\left(\dot{\gamma}_{\mathrm{Cr}} \not 2000 \mathrm{~s}^{-1}\right.$ pour ces deux composites), le mode d'endomagement dans les échantilions est une fissuration dans les couches de tissu et un délaminage entre deux couches de tissu. Ceci explique pourquoi les contraintes maximales sont décroissantes pour les courbes citées antérieurement : $n^{\circ} 5$ (type I) $n^{\circ} 5,7$ (type II) et $n^{\circ} 5,7$ (type III) sur la figure $6 ; n^{\circ} 3$ sur la figure 8 .

\section{DISCUSSION ET CONCLUSION}

A l'issue des premiers résultats expérimentaux, il apparaît que les paramètres tels que la vitesse de déformation $\dot{\gamma}$, la température des essais $\mathbf{m}$, l'élévation de tempé- 


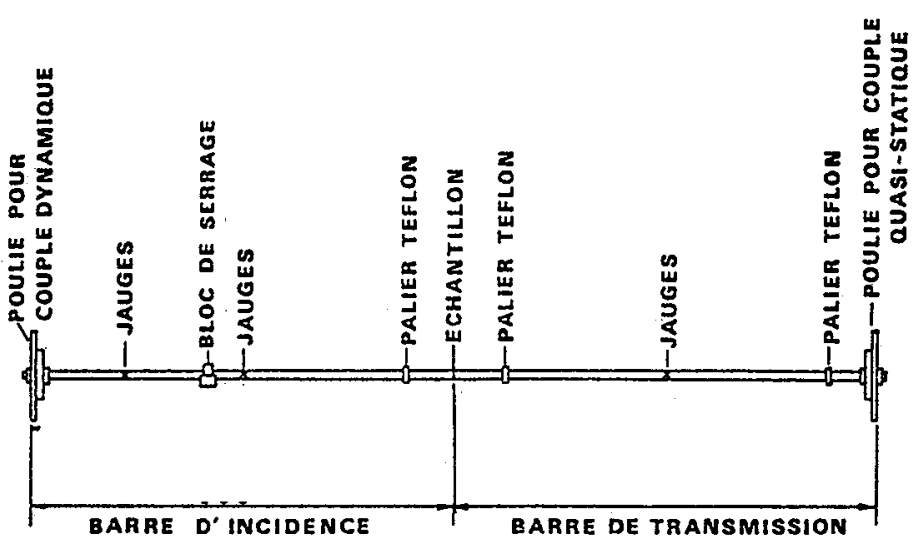

Fig. 1 - Barre de Kolsky modifiée pour la torsion

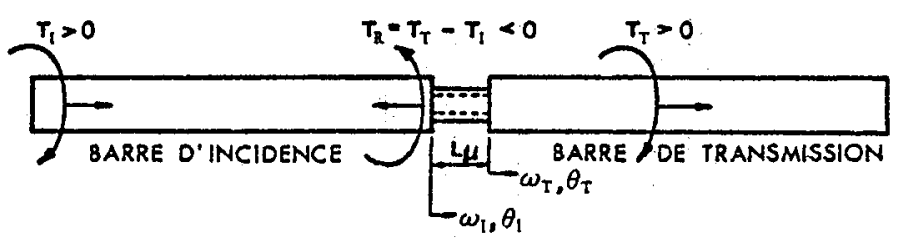

Fig. 2 - Relation entre les couples de torsion

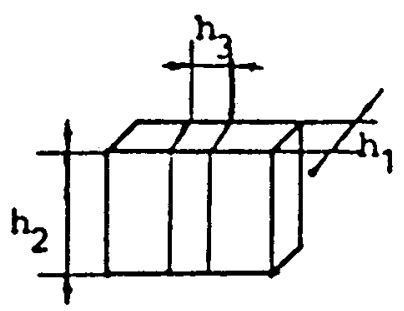

Fig. 3.a - Echantilion non entalllé

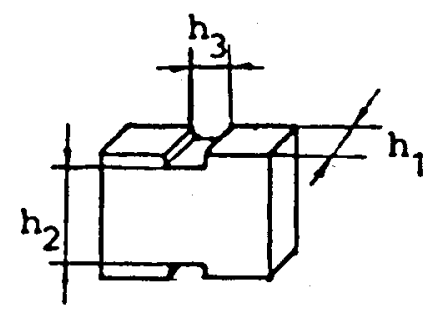

Fig. 3.b - Echantilion entaillé 
Tableau 1 : géanétrie des échantillons

\begin{tabular}{|c|c|c|c|c|}
\hline Echantillons & Type & $h_{1}(\mathrm{~mm})$ & $h_{2}(\mathrm{~mm})$ & $h_{3}(\mathrm{~mm})$ \\
\hline non-entaillés & I & 3,2 & 10,8 & 2,8 \\
\hline \multirow{2}{*}{ entaillés } & II & 3,2 & 8,8 & 3,2 \\
\cline { 2 - 5 } & III & 3,2 & 6,8 & 3,2 \\
\hline
\end{tabular}

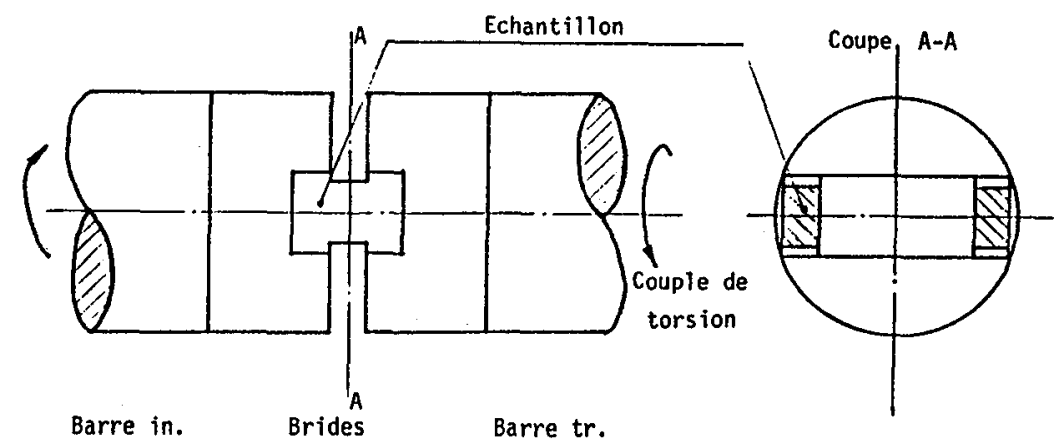

Fig. 4 - Encastrement des échantillons parallélépipédiques 

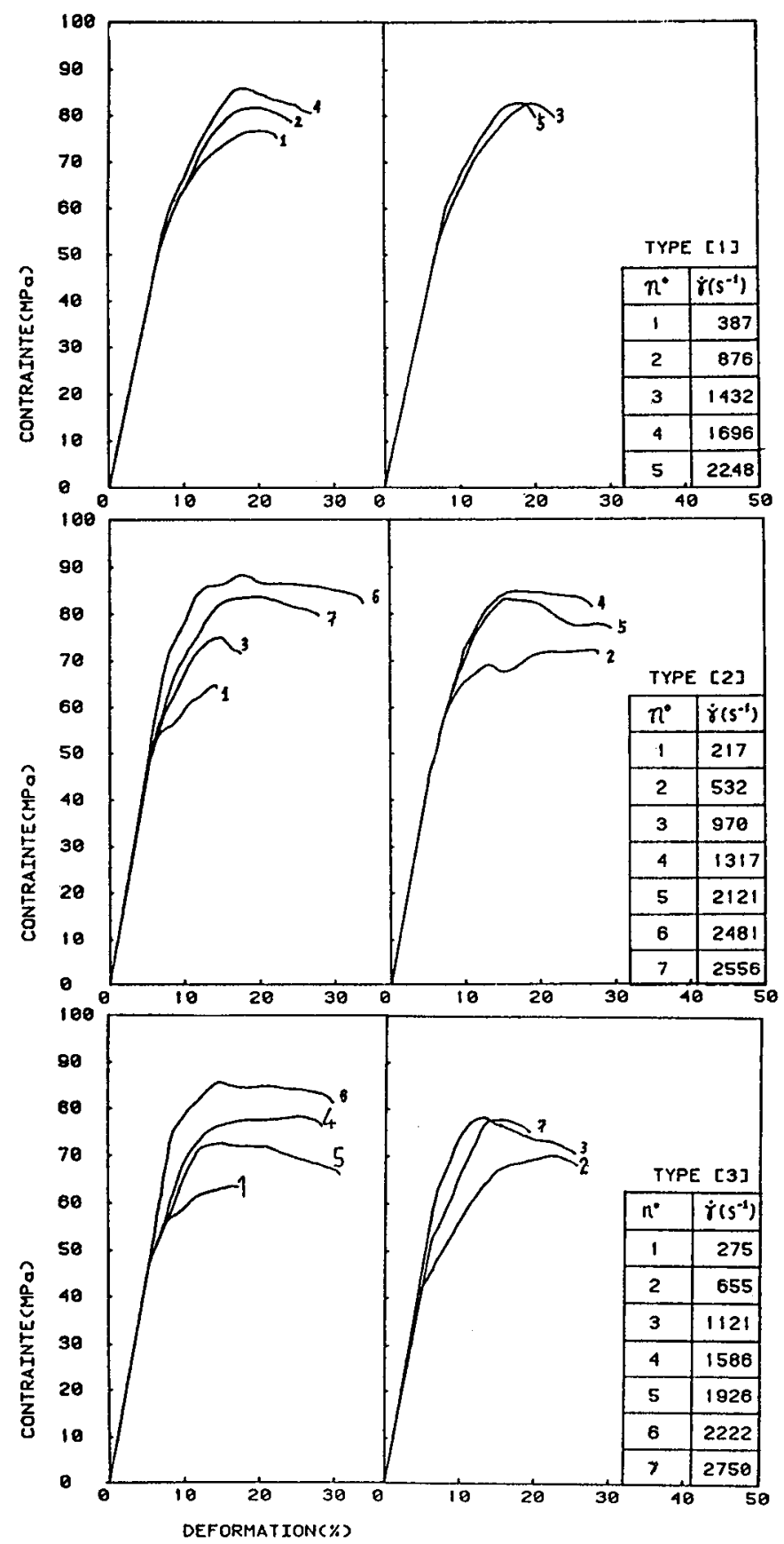

Fig. 5 - Courbes de contrainte-déformation, composite I. 


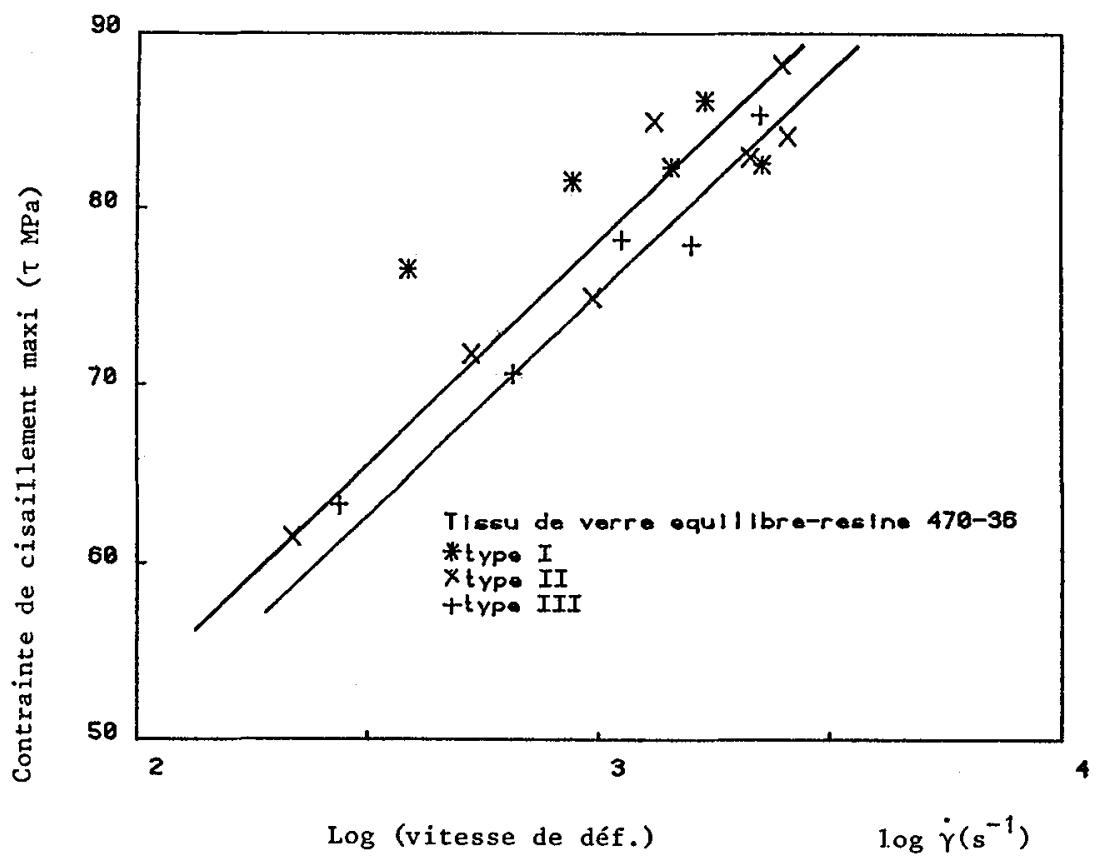

Fig. 6 - Influence de la vitesse de déformation sur la contrainte de cisaillement maximale.

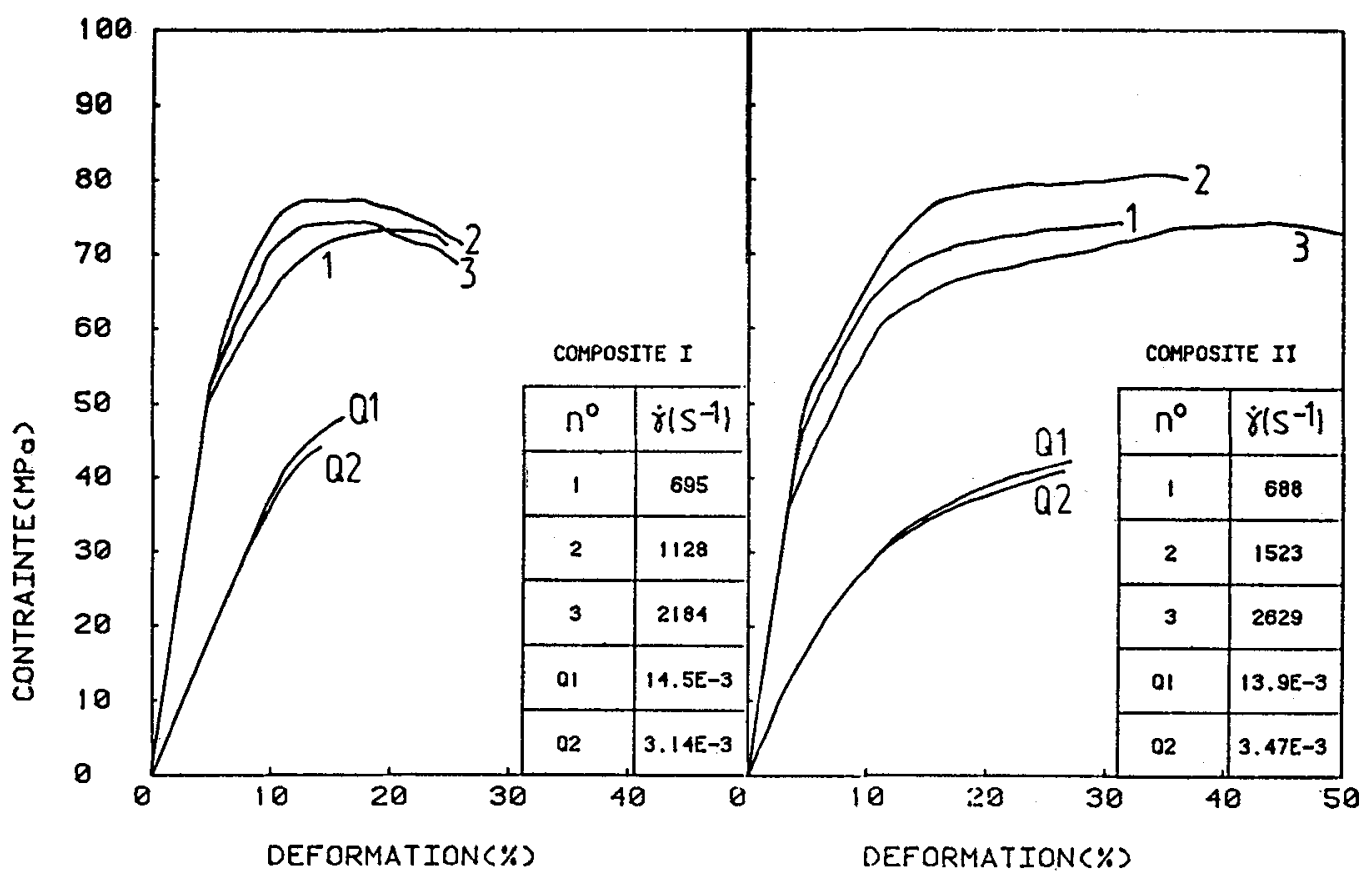

Fig. 7 - Courbes contrainte-déformation moyennes 
Tableau 2 : Endormagement représentatif

\begin{tabular}{|c|c|c|c|c|c|}
\hline \multirow{2}{*}{\multicolumn{2}{|c|}{ Mode de sollicitation }} & \multicolumn{2}{|c|}{ Déformation $(q)$} & \multirow{2}{*}{$\begin{array}{l}\text { Endormagement } \\
\text { représentatif }\end{array}$} & \multirow{2}{*}{ Allure d'endommagement } \\
\hline & & $\begin{array}{l}\text { Compo- } \\
\text { site I }\end{array}$ & $\begin{array}{l}\text { Compo- } \\
\text { site II }\end{array}$ & & \\
\hline \multicolumn{2}{|c|}{ quasi-statique } & $10 \sim 20$ & $20 \sim 30$ & & très faible fissuration \\
\hline \multirow{3}{*}{ 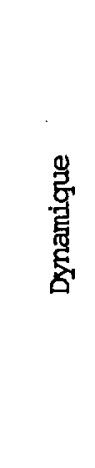 } & $\dot{\gamma}$ faible & \multirow{3}{*}{$30 \sim 40$} & \multirow{3}{*}{30,50} & & faible fissuration \\
\hline & $\begin{array}{c}\dot{\gamma} \text { intermé- } \\
\text { diaire }\end{array}$ & & & & fissuration importante \\
\hline & $\begin{array}{l}\dot{\gamma} \text { tres impor- } \\
\text { tante } \\
\left(\dot{\gamma}>\dot{\gamma}_{c r}\right)\end{array}$ & & & & $\begin{array}{l}\text { fissuration et } \\
\text { delaminage }\end{array}$ \\
\hline
\end{tabular}

rature $\Delta \mathrm{T}$ en cours de déformation plasticque de la résine et le cisaillement interlamellaire sont prédominants dans le comportement aux chocs des matériaux composites. Nous proposons qu'un modẻle général devrait être mis sous la forme :

$\tau=A \ln \dot{\gamma}+B \dot{f}(T, \Delta T)+\Phi$ où $A$ et $B$ sont des constantes; $\Phi$ une fonction de tous les autres paramètres physiques, chimiques et mécaniques. Au-dessous de $\dot{\gamma}_{\mathrm{Cr}}{ }^{\prime}$ les influences de $B f(T, \Delta T)$ et de $\Phi$ sont négligeables devant $A$ ln $\dot{\gamma}$. Il est très difficile actuellement de détexminer quantitativement l'influence de la vitesse de sollicitation. Il faut egalement noter que les essais ne sont pas reproductibles exactement et que la dispersion des valeurs impose de faire de nombreux essais pour quantifier le phénomene statistiquement.

Cette méthodologie s'adapte bien à l'étude du phénomène de décohésion localisée (fissuration et délaminage) car il est possible de relier les phénomènes d'endommagement du matériau à ses propriétés mécaniques. Ceci nous conđuira à tester des composites à fibres unidirectionnelles de verre et les résines pures. Jes résultats nous permettront de bien comprendre les mécanismes tels que le iélaminage, la décohésion des fibres, les fissures translaminaires, et de proposer des modèles pratiques applicables au calcul des structures.

\section{REFERENCES}

/1/ F. Devries et F. Lene, Rapport G.R.E.C.O. n¹15 (1984).

/2/ C.Y. Chiem, J.P. Ramousse et R. CoziC, Convention E.N.S.M./C.E.T.I.M., Rapport $\mathrm{n}^{\circ} \mathrm{CRC} / 8407-1 \quad(1984)$.

/3/ J. Duffy, J.D. Campbell and R.H. Hawley, J. of Applied Mechanics (Mars 1971), pp. 83-91.

14/ C.Y. Chiem, Thèse de Docteur-ès-Sciences Etat (Juin 1980).

/5/ C.Y. Chiem, P. Blinot, R. Cozic, J.P. Dubois, Rapport Technique nºMI-83-X15 (Mai 1983).

16/ C.Y. Chiem et J. Duffy, J. Mat. Sci. and Eng., vol. 57 (1982), n², pp. 233-247. 